\title{
Dynamique et structure de la flore de la Savane Protégée des Feux dans la Réserve Scientifique de Lamto (Centre de la Côte d'Ivoire)
}

\author{
Gnahoré Eric, $M A$ \\ Missa Koffi, PhD \\ Koné Moussa, PhD \\ Gueulou Nina, MA \\ Bakayoko Adama, PhD
}

UFR des Sciences de la Nature, Université Nangui Abrogoua, Côte d'Ivoire

Doi: 10.19044/esj.2018.v14n36p432 URL:http://dx.doi.org/10.19044/esj.2018.v14n36p432

\begin{abstract}
This work that is one of the first of its kind in Unburned Savannah in Lamto, center of Ivory Coast, intends to characterize the vegetation in terms of dynamic evolution and plant structure, 53 years after its protection from fires. Surfaces plot, linear surveys and itinerant methods were used to collect floristic data. The diversity and the wealth of flora, as well as the structure of the different types of vegetation has been analyzed. 176 plant species belonged to genera 143 kinds and 49 families were indentified. The most important families are the Leguminosae $(14.77 \%)$, Rubiaceae $(13.63 \%)$ and Apocynaceae $(10.79 \%$ ). About $15.37 \%$ (27 species) of this flora represents the particular statute species. Among the inventoried species, 11 are exploitable species or exploited as timber of which ten are forest species. Due to richnes of its flore and flora dynamic, the Unburned Savannah must be protected. Based on an ascending hierarchical classification performed by using 11 linear surveys, this study has demonstrated the existence of a floristic variability to three groups.
\end{abstract}

Keywords: Phytodiversity, vegetation, plant communities, Côte d'Ivoire

\section{Resume}

Ce présent travail qui est l'un des premiers du genre dans la Savane Protégée des Feux à Lamto centre de la Côte d'Ivoire, se propose la caractérisation de la végétation en termes d'évolution de la dynamique et de structure végétale, 53 années après sa protection des feux. La collecte des données sur la flore a combiné les méthodes de relevé de surface, de relevé 
linéaire et de relevé itinérant. Une analyse des données collectées sur le terrain a été effectuée autour de la diversité et la richesse de la flore, mais également de la structure des différents peuplements. Au total, 176 espèces végétales reparties en 143 genres et 49 familles ont été identifiées. Les familles les plus représentées sont les Leguminosae (14,77\%), les Rubiaceae $(13,63 \%)$ et les Apocynaceae $(10,79 \%)$. Environ 27 espèces $(15,34 \%)$ de cette flore représentent des espèces à statut particulier. Parmi les espèces inventoriées, 11 sont des espèces exploitables ou exploitées comme bois d'œuvre dont 10 sont des espèces forestières. Cette étude a donc permis de mettre en évidence la richesse et la dynamique de la flore de la Savane Protégée des Feux. Sur la base d'une classification hiérarchique ascendante effectuée à l'aide des 11 relevés linéaires, cette étude a permis de montrer l'existence d'une variabilité floristique de trois groupes.

Mots-clés: Phytodiversité, végétation, groupements végétaux, Côte d'Ivoire

\section{Introduction}

Le terme «savane» est employé pour désigner des écosystèmes naturels où coexistent une strate herbacée continue et une strate ligneuse plus ou moins discontinue (Menaut, 1983). Leurs structures et leurs dynamiques représentent donc des enjeux majeurs dans un contexte de changement climatique et d'érosion de la biodiversité. Une des menaces à leur conservation les plus importantes constitue leur transformation par un processus rapide de colonisation par les espèces ligneuses, qui tend à transformer ces écosystèmes ouverts en forêts (Demers-Lemay, 2014). Au cours du dernier siècle, une colonisation des milieux ouverts par les espèces ligneuses a été rapportée partout sur la planète (Eldridge et al., 2011). Tout comme de nombreuses savanes tropicales, la Savane Protégée des Feux (SPF) dans la Réserve Scientifique de Lamto n'est pas en marge de cette évolution.

La transformation des savanes peut être relativement rapide: certaines d'entre elles sont remplacées par des forêts en moins de 40 ans (Bond \& Parr, 2010). C'est le cas de la SPF protégée depuis 1962. Cette colonisation ligneuse résulterait d'une combinaison d'absence de facteurs d'origine anthropique ayant modifié les relations de compétition pour les ressources entre les strates herbacées et ligneuses (Estell et al., 2012 ; Brunelle et al., 2013).

Hormis les travaux effectués en 1984 par Devineau et al. (1984), cette formation végétale protégée du feu est très mal connu au niveau de la dynamique de l'évolution de la flore, du couvert végétal et de structure d'où cette étude. L'objectif général de la présente étude est de contribuer à la conservation de la SPF. De façon spécifique, il s'agit de comprendre la dynamique de la végétation par l'évaluation des variations floristique et structurale. 


\section{Matériel et Méthodes \\ Site d'étude}

La Réserve Scientifique de Lamto est située dans la partie centrale de la Côte d'Ivoire (Afrique de l'ouest), à la pointe du "V baoulé" entre la savane guinéenne et la forêt semi décidue. Elle se localise dans la région de l'AgnébyTiassa, dans le département de Tiassalé, à environ $165 \mathrm{Km}$ au Nord-Ouest d'Abidjan, entre les $6^{\circ} 13^{\prime}$ et $6^{\circ} 25^{\prime}$ latitude Nord et les $4^{\circ} 97^{\prime}$ 'et $5^{\circ} 02^{\prime}$ ' longitude Ouest. Elle a une superficie de 2500 hectares (Vuattoux et al., 1998). (Figure 1). A l'intérieur de cette Réserve Scientifique se situe la Savane Protégée des Feux.

Une parcelle de savane arbustive à Andropogon située en haut de pente, de quelques dizaines d'hectares, a été protégée du feu depuis 1962 et n'a brûlée accidentellement qu'une seule fois. L'absence prolongée des feux rompt l'équilibre apparent des savanes, comme le montre l'évolution des zones protégées des feux aux environs immédiats de la station d'écologie de Lamto. Les espèces de savane se développent d'abord et atteignent des tailles plus élevées qu'en savane brûlée. Des espèces sensibles au feu s'installent ensuite et prolifèrent avec une proportion considérable de plantes lianescentes. L'évolution de la végétation a été rapide et profonde (Vuattoux, 1970). On observe un accroissement du nombre d'espèces d'arbres et de la taille de chaque individu des ligneux. Elle se traduit par un envahissement par des arbres de forêt et de nombreuses espèces lianescentes. Les espèces de savane disparaissent alors peu à peu et le milieu devient une forêt secondaire. La présence de rôniers (Borassus aethiopum Mart) vivants ou morts, des plaques herbeuses et de quelques espèces de ligneux de savane témoigne du passé savanicole de ce milieu.

Sur la base du «bilan climatique», la Réserve Scientifique de Lamto possède quatre saisons déterminées par le régime des pluies (Pagney, 1988). Il s'agit d'une grande saison des pluies (d'avril à juillet), d'une petite saison sèche (en août), d'une petite saison des pluies (de septembre à octobre) et une grande saison sèche (de novembre à février). La pluviométrie moyenne annuelle observée dans ces dernières décennies était de 100,75 mm avec une température moyenne annuelle de $28,27^{\circ} \mathrm{C}$.

Les sols de Lamto ont été étudiés du point de vue strictement pédologique pour en déterminer la valeur agronomique (Riou, 1964 ; Delmas, 1967). On peut avec ceux-ci distinguer quatre grands types de sols : sols ferrugineux tropicaux de plateau et de haut de pente, les sols ferrugineux de pente, les sols hydromorphes de bas de pente et les terres noires sur amphibolites.

La végétation de la Réserve Scientifique de Lamto est une mosaïque de forêt-savane, constituée par des galeries forestières riveraines du fleuve 
Bandaman, des lambeaux de forêts denses semi décidues et une savane à rônier (Devineau, 1975).

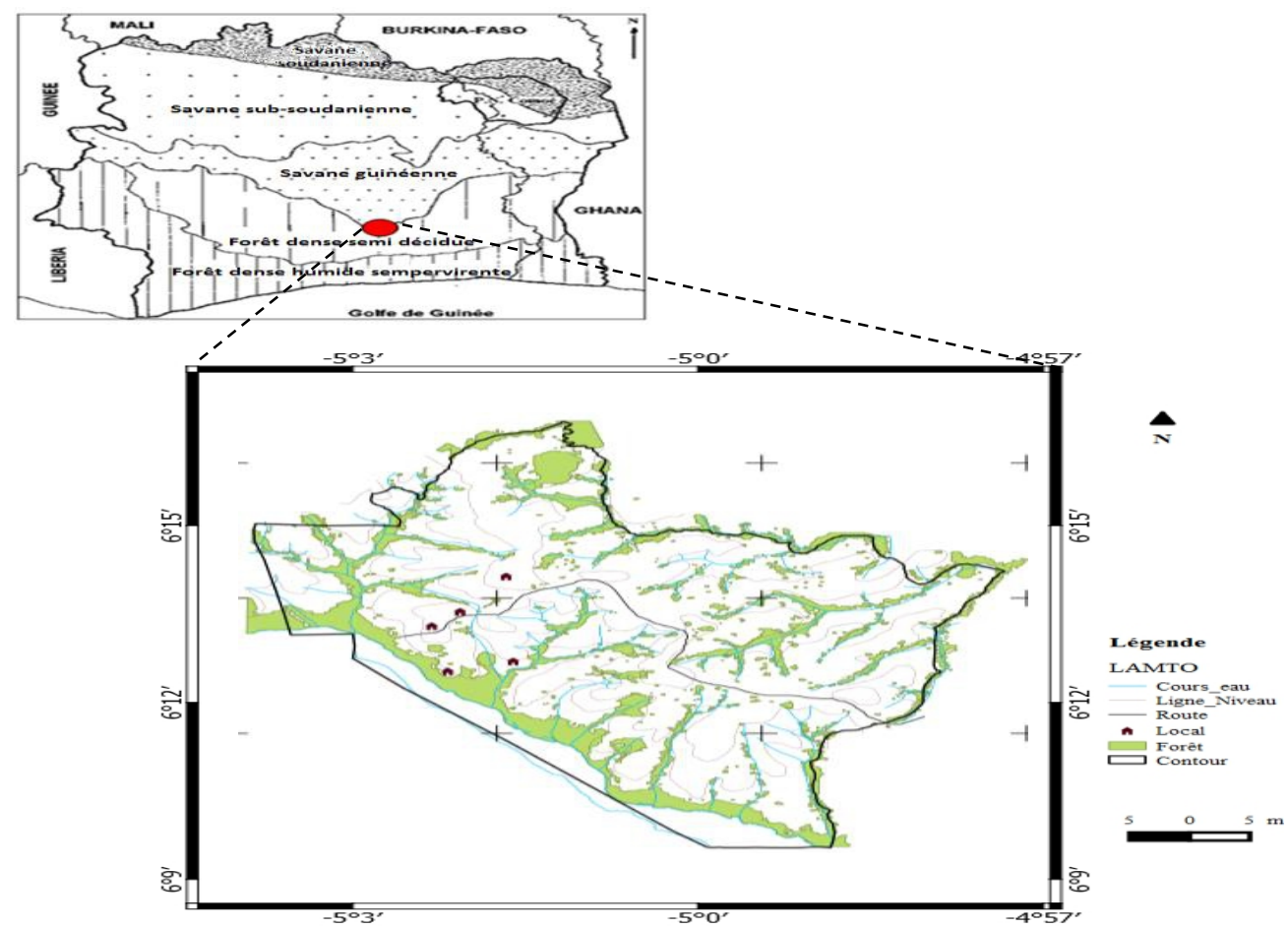

Figure 1: Localisation de la zone d'étude

\section{Méthode de terrain}

Une combinaison de trois méthodes d'échantillonnages a été mise en œuvre pour l'inventaire de la flore de la Savane Protégée des Feux (SPF). Il s'agit de relevés linéaires, de relevés de surface et itinérants. La méthode des relevés linéaires a été proposée par (Gautier et al., 1994). Elle a déjà été utilisée par (Chatelain, 1996 ; Missa et al., 2015). Elle consiste à tendre horizontalement, à ras du sol, une corde longue de 200 mètres. A partir de ce dispositif, nous avons effectué 100 mesures à des intervalles de deux mètres et cela grâce à quatre jalons emboitables de $2 \mathrm{~m}$ de hauteur chacun, placé verticalement à chaque point. Les mesures consistent à relever tous les contacts entre le piquet vertical et la végétation, sans limite de hauteur. Audelà de huit mètres, les mesures sont faites par simple estimation. Pour chaque contact du jalon avec la corde, on identifie toutes les espèces touchant le jalon, et on prend en compte, leurs hauteurs minimales et maximales de contact avec celui-ci.

Concernant la méthode des relevés de surface, c'est une méthode classique déjà utilisées par plusieurs auteurs (Gueulou et al., 2018 ; Missa et 
al., 2015 ; Bakayoko, 1999) et dans plusieurs milieux. L'inventaire floristique s'est déroulé en trois grandes étapes. Pour cette étude, une surface de $400 \mathrm{~m}^{2}$ a été choisie, soit, 20 mètres sur 20 mètres. Chaque parcelle d'étude a été subdivisée en quatre sous-parcelles. A l'intérieur de la formation végétale six parcelles carrées de 20 mètres de côté au total ont été mise en place. En effet, la mesure de la circonférence des espèces ligneuses s'est effectuée à 1,30 m au-dessus du sol avec un diamètre à hauteur de poitrine supérieur $(\mathrm{DBH})$ ou égal à $5 \mathrm{~cm}$. Un arbre peut être constitué de plusieurs brins, ceux-ci sont comptabilisés séparément si la séparation est en dessous de 1,30 m du sol.

La deuxième étape consiste à délimiter à l'intérieur de chaque parcelle une sous-parcelle de superficie de $5 \mathrm{~m}$ x $5 \mathrm{~m}$ soit un carré $25 \mathrm{~m}^{2}$ appelée parcelle de régénération. Dans celle-ci toutes les espèces végétales (arbustives, lianescentes, ou herbacées) y ont été inventoriées sans tenir compte de la mesure du DBH.

Les informations recueillies à l'aide des deux premières méthodes ont été complétés par la méthode du relevé itinérant. Elle consiste à parcourir chaque parcelle dans toutes les directions en notant toutes les nouvelles espèces croisées au hasard qui n’ont pas encore été inventoriées (Adou et al., 2011). La liste floristique de la SPF a été établie avec les espèces issues des relevés de surface, des relevés itinérants et des relevés linéaires. La nomenclature s'est basée sur les travaux de (Hutchinson \& Dalziel, 1954) et confirmée par la dernière classification phylogénétique (APG IV, 2016). Pour la désignation des types morphologiques et biologiques, nous nous sommes basés sur les travaux d'Aké-Assi (2002).

\section{Analyse des données}

Les données collectées ont servi de base aux analyses de la flore et de la structure des peuplements. Ainsi, nous nous sommes intéressés à l'analyse de la liste générale (richesse) de la flore de la Savane Protégée des Feux (SPF) en mettant en évidence les espèces caractéristiques, les espèces à statut particulier. Il s'agit des espèces endémiques, rares et menacées d'extinction et les espèces exploitables ou exploitées comme bois d'œuvre.

L'indice de diversité de Shannon-Weaver (H') (Shannon \& Weaver, 1949) a permis de quantifier la diversité floristique de l'ensemble de la SPF. Cet indice mesure également l'entropie du système sur la base des proportions observées. Il a l'avantage de tenir compte du nombre d'espèces et de l'abondance des espèces. Il est souvent utilisé pour exprimer la diversité des relevés (Vroh et al., 2010). Cet indice a été calculé avec le logiciel EstimateSWin800.

$$
\mathrm{H}^{\prime}=-\sum_{i=1}^{n} P_{i} * \log _{2}\left(\mathbf{P}_{i}\right)
$$

$\operatorname{avec} \mathbf{P}_{\mathbf{i}}=\frac{\boldsymbol{n}_{\boldsymbol{i}}}{\boldsymbol{N}}$ 
ni: nombre d'individus d'une espèce donnée, i allant de 1 à $\mathrm{S}$ (nombre total d'espèces).

$\mathbf{N}$ : nombre total d'individus de toutes les espèces confondus dans le milieu.

$\mathbf{P}_{\mathbf{i}}=$ proportion de l'espèce.

De l'indice de diversité de Shannon-Weaver (H') a été déduit par la suite, l'indice de l'équitabilité (E) (Piélou, 1966).qui renseigne sur la répartition des effectifs entre les différentes espèces (Barbault, 1982). Il varie de 0 à 1 . Il tend vers 0 quand une seule espèce est abondante et tend vers 1 quand les individus sont régulièrement repartis entre les espèces. Cet indice est calculé à partir de la formule suivante:

$\mathbf{E}=\frac{\boldsymbol{H}^{\prime}}{\log _{2} \boldsymbol{S}} \quad$ avec $\mathbf{S}:$ le nombre total d'espèces (la richesse spécifique).

La structure verticale des peuplements a été décrite et les hauteurs des différentes espèces sont comparées entre elles. Au niveau de la structure horizontale, notre analyse a portée sur le calcul de recouvrement. Sur la base des groupes formés à l'aide du logiciel STATISTICA version 7.1, nous avons extraire les cortèges d'espèces qui caractérisent les différents groupes. On obtient un support visuel permettant une approche de la physionomie de la végétation rencontrée et une idée de la répartition spatiale des espèces qui la composent. Elle permet aussi d'aborder, dans la direction verticale, la stratification de la végétation et dans la direction horizontale, l'état de répartition des espèces, notamment l'homogénéité ou l'hétérogénéité de la végétation. Dans cette étude, le recouvrement des espèces est approché par le pourcentage des points de contact des espèces avec le jalon placé verticalement. Il concerne le niveau spécifique et est exprimé par la fréquence à laquelle l'espèce est rencontrée le long du relevé. Il concerne également la répartition verticale de la végétation et correspond à la distribution de toutes les espèces confondues dans les intervalles de hauteur, exprimé ici par le pourcentage. Les intervalles de hauteur $0<$ à $\leq 2 \mathrm{~m}, 2<$ à $\leq 4 \mathrm{~m}, 4<$ à $\leq 8 \mathrm{~m}$, $8<$ à $\leq 16 \mathrm{~m}, 16<\mathrm{à} \leq 32 \mathrm{~m}$ et $>32 \mathrm{~m}$ ont été utilisés (Chatelain, 1996).

Pour mesurer la similarité ou dissimilarité floristique entre les différents relevés, les méthodes de la classification hiérarchique $(\mathrm{CAH})$ et de l'analyse en composante principale $(\mathrm{ACP})$ ont été réalisées à l'aide du logiciel STATISTICA version 7.1.

\section{Résultats}

\section{Etude floristique}

La compilation des listes d'espèces inventoriées à travers les 11 relevés linéaires, les six relevés de surface et des relevés itinérants a permis d'établir une liste de 176 espèces ( 77 espèces pour les relevés de surface et itinérant et 156 pour les relevés linéaires et itinérant) reparties en 143 genres et 49 
familles. Cette étude révèle trois grandes familles qui sont les plus dominantes en espèces végétales. Ce sont les Leguminosae (26 espèces), les Rubiaceae (24 espèces) et les Apocynaceae (19 espèces) (Figure 2). On note que l'essentiel de la flore inventoriée est représentatif des taxons des régions Guinéo-Congolaise (GC) $(60,79 \%)$, Guinéo-Congolaise et SoudanoZambèzienne (GC-SZ) (31,25 \%), Guinéo-Congolaise Ouest Africaine (GCW) (4,54 \%), Soudano-Zambèzienne (SZ) (2,84 \%) et endémique ivoirienne (GCI) (0,56\%) (Figure 3).

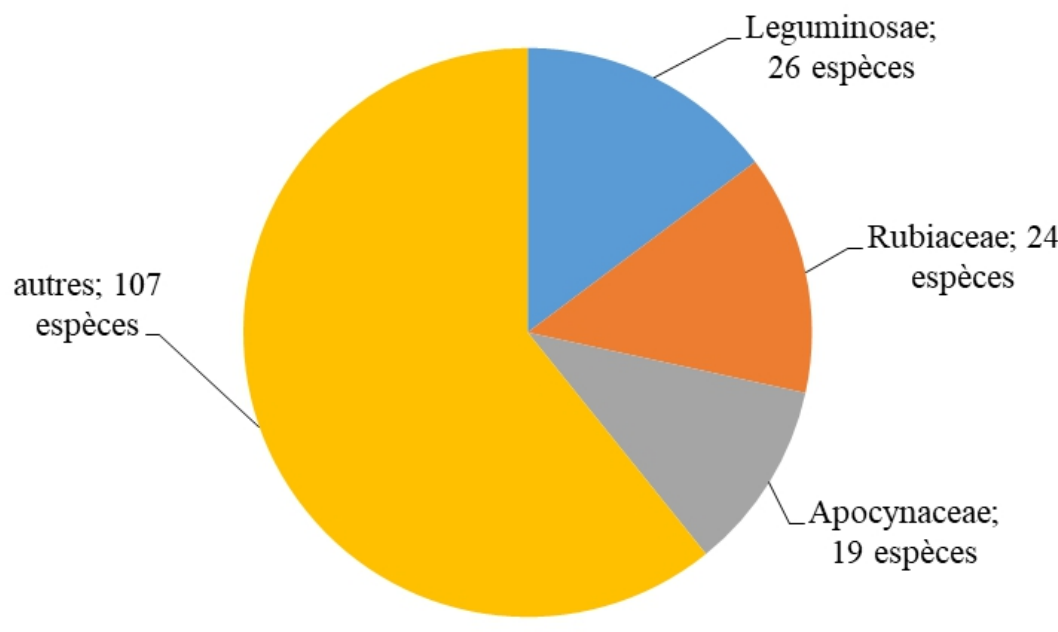

Figure 2 : Spectre des familles dominantes dans la Savane Protégée des Feux (SPF)

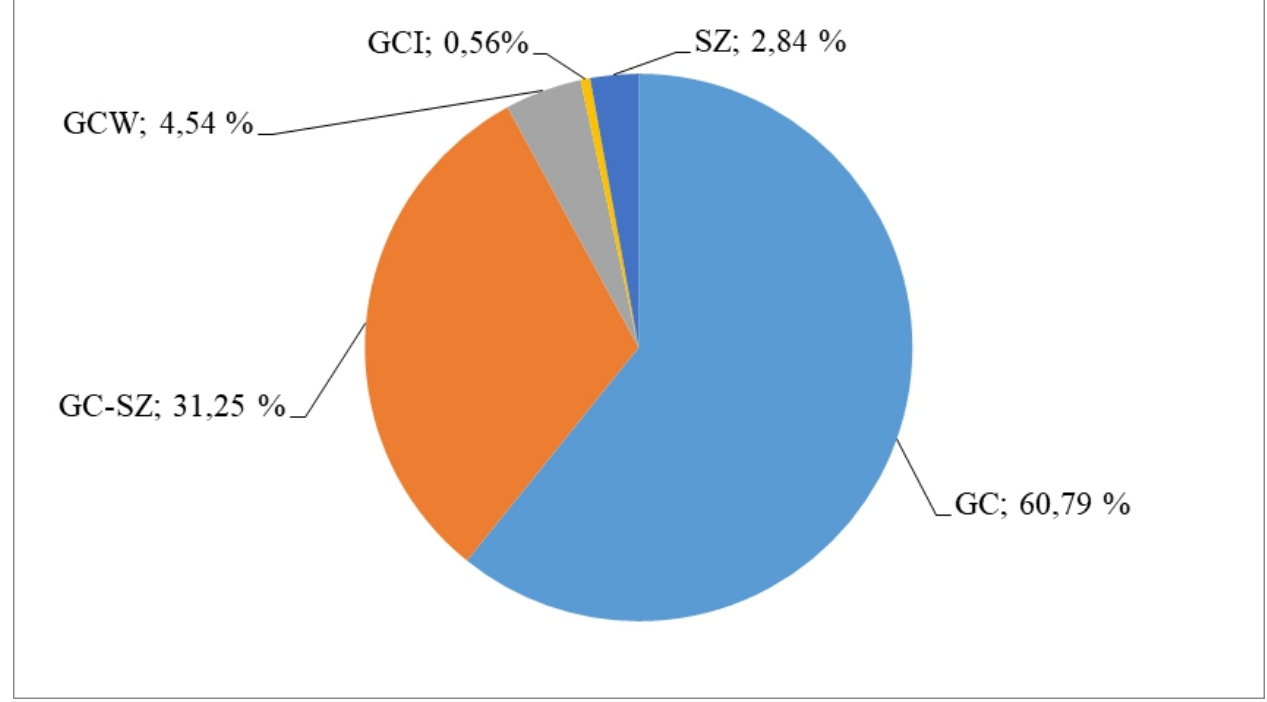

Figure 3 : Répartition chorologique des espèces inventoriées dans la SPF 
Neuf espèces inventoriées sont reconnues comme endémiques du bloc forestier de l'Afrique de l'ouest (GCW) avec Leptoderris meigei Aké-Assi \& Mangenot strictement endémique de la Côte d'Ivoire (GCI) (Tableau 1).

Selon 1'UICN (2018), parmi ces espèces inventoriées on note la présence de deux espèces sont vulnérables (VU), 13 à préoccupation mineure (LC), une à donnée insuffisante (DD) et deux à risque faible / quasi menacée (NT) (Tableau 2).

Tableau 1 : Liste des espèces relevées dans la SPF reconnue comme endémiques

\begin{tabular}{|l|l|l|}
\hline Espèces & Familles & Statuts \\
\hline Adenia dinklagei Hutch. \& Dalziel & Passifloraceae & GCW \\
\hline Aristolochia embergeri Nozeran \& N.Hall & Aristolochiaceae & GCW \\
\hline Cola caricifolia (G.Don) K.Schum. & Malvaceae & GCW \\
\hline Cola cordifolia (Cav.) R.Br. & Malvaceae & GCW \\
\hline Kolobopetalum leonense Hutch. \& Dalziel & Menispermaceae & GCW \\
\hline Landolphia membranacea (Stapf) Pichon & Apocynaceae & GCW \\
\hline Leptoderris miegei Aké-Assi \& Mangenot & Leguminosae & GCI \\
\hline Strychnos dinklagei Gilg & Loganiaceae & GCW \\
\hline Tiliacora dinklagei Engl & Menispermaceae & GCW \\
\hline
\end{tabular}

Tableau 2 : Liste des espèces vulnérables (VU), à préoccupation mineure (LC), à donnée insuffisante (DD) et risque faible / quasi menacée (NT) présentes de la SPF

\begin{tabular}{|c|c|c|}
\hline Espèces & Familles & Statuts \\
\hline Afzelia africana Pers. & Leguminosae & VU A1d ver 2.3 \\
\hline Albizia adianthifolia (Schum.) W.Wight & Leguminosae & LC ver 3.1 \\
\hline Albizia ferruginea (Guill. \& Perr.) Benth & Leguminosae & VU A1cd ver 2.3 \\
\hline Andira inermis (Wright) DC. & Leguminosae & LC ver 3.1 \\
\hline Baphia nitida Lodd. & Leguminosae & LC ver 3.1 \\
\hline Borassus aethiopum Mart. & Arecaceae & LC ver 3.1 \\
\hline Ceiba pentandra (L.) Gaertn. & Malvaceae & LC ver 3.1 \\
\hline Celtis zenkeri Engl. & Cannabaceae & LC ver 3.1 \\
\hline Coffea canephora Pierre ex A.Froehner & Rubiaceae & LC ver 3.1 \\
\hline Commelina erecta $(\mathrm{L})$. & Commelinaceae & LC ver 3.1 \\
\hline Elaeis guineensis Jacq. & Arecaceae & LC ver 3.1 \\
\hline Mangifera indica $\mathrm{L}$. & Anacardiaceae & DD ver 2.3 \\
\hline Millettia zechiana Harms & Leguminosae & LC ver 3.1 \\
\hline Milicia excelsa (Welw.) C.C.Berg & Moraceae & NT ver 2.3 \\
\hline Pouteria altissima (A.Chev.) Baehni & Sapotaceae & NT ver 2.3 \\
\hline Senna siamea (Lam.) H.S.Irwin \& Barneby & Leguminosae & LC ver 3.1 \\
\hline Spathodea campanulata P.Beauv. & Bignoniaceae & LC ver 3.1 \\
\hline Vitex doniana Sweet & Lamiaceae & LC ver 3.1 \\
\hline
\end{tabular}

La flore de la SPF présente également une importante valeur économique. Parmi ces espèces inventoriées, 11 sont des essences forestières 
exploitées ou exploitables comme bois d'œuvres (Tableau 3). Ces essences forestières ont été subdivisées en trois catégories sur la base du critère de commercialisation en Côte d'Ivoire. Les essences principales couramment commercialisées (catégorie 1) sont au nombre de quatre, celles qui sont sporadiquement commercialisées (catégorie 2) sont également au nombre de cinq et celles qui sont à promouvoir (catégorie 3) au nombre de deux.

Tableau 3 : Liste des essences relevées dans la SPF et identifiées comme exploitables ou exploitées en tant que bois d'œuvres en Côte d'Ivoire

\begin{tabular}{|l|l|l|l|}
\hline $\mathbf{N}^{\circ}$ & Espèces & Familles & Catégories \\
\hline 1 & Afzelia africana & Leguminosae & 1 \\
\hline 2 & Albizia adianthifolia & Leguminosae & 2 \\
\hline 3 & Albizia ferruginea & Leguminosae & 2 \\
\hline 4 & Antiaris toxicaria & Moraceae & 1 \\
\hline 5 & Berlinia grandiflora & Leguminosae & 2 \\
\hline 6 & Celtis zenkeri & Ulmaceae & 3 \\
\hline 7 & Ceiba pentandra & Malvaceae & 1 \\
\hline 8 & Funtumia africana & Apocynaceae & 2 \\
\hline 9 & Mitragyna inermis & Rubiaceae & 2 \\
\hline 10 & Morus mesozygia & Moraceae & 1 \\
\hline 11 & Sterculia tragacantha & Malvaceae & 3 \\
\hline
\end{tabular}

Concernant les types biologiques, sur les 176 espèces végétales inventoriées 164 sont des espèces phanérophytes soit 93,18 \% des types biologiques qui peuplent la SPF. Les espèces se répartissent en 105 espèces (soit 59,65\%) (ligneuses et lianescentes) microphanérophytes, 31 espèces (soit 17,61\%) (ligneuses et lianescentes) mésophanérophytes, 21 espèces (soit $11,93 \%$ ) (ligneuses et lianescentes) nanophanérophytes, quatre espèces (soit $2,27 \%$ ) chaméphytes, 2 espèces (soit 1,13\%) géophytes, deux espèces (soit $1,13 \%$ ), géophyte rhizomateux, cinq espèces (soit 2,84 \%) mégaphanérophytes, une espèce (soit $0,56 \%$ ) épiphyte et une espèce (soit 0,56 $\%$ ) épiphyte herbeuse (figure 4). Les microphanérophytes sont les plus prépondérantes suivi des mésophanérophytes. 


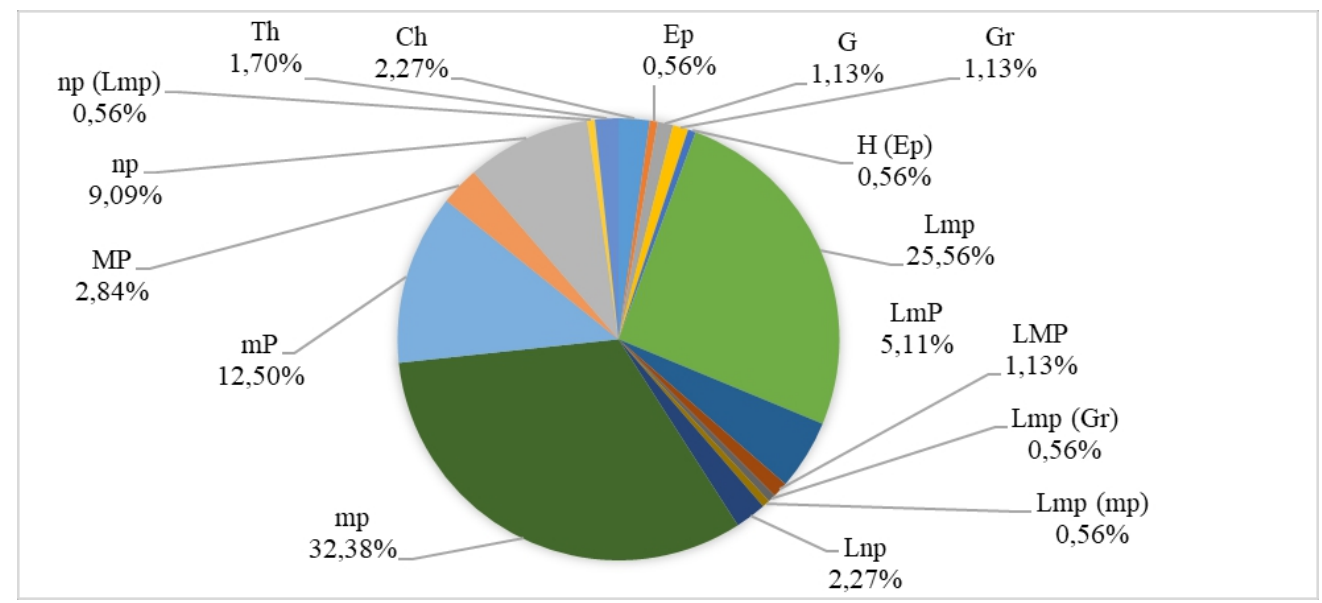

Figure 4 : Spectre des types biologiques des espèces inventoriées dans la SPF

Les espèces recensées dans la SPF comportent une grande proportion d'espèces d'arbustes $(42,04 \%)$ soit 74 espèces. Les espèces arborescentes font $14,74 \%$ soit 26 espèces. En ce qui concerne la proportion des espèces lianescentes et des espèces herbacées, nous avons respectivement 35, $22 \%$ soit 64 espèces pour les lianes et $9,09 \%$ soit 16 espèces pour les herbes (Figure 5).

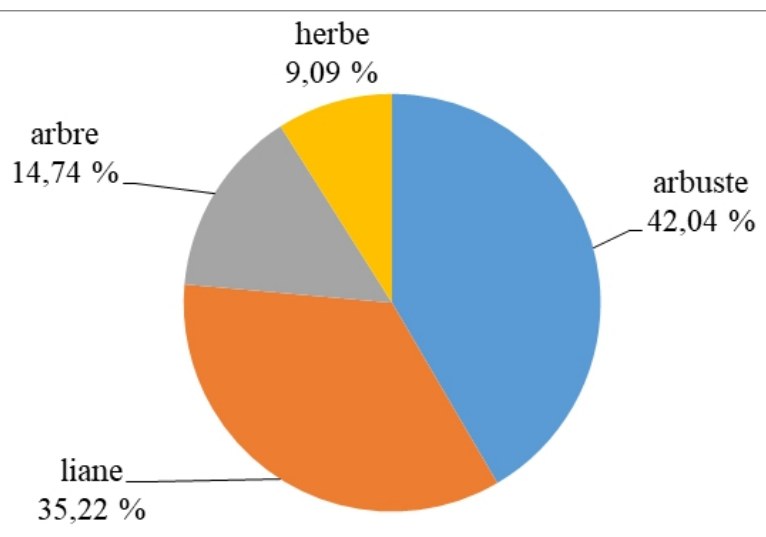

Figure 5 : Spectre des types morphologiques des espèces inventoriées dans la SPF

Le calcul de l'indice de diversité moyen de Shannon-Weaver à partir de l'abondance des individus est de 1,217. Cet indice est variable au niveau des différents relevés effectués. L'indice de diversité moyen de Piélou est 0,765 pour l'ensemble de tous les relevés.

\section{Structure de la végétation}

Nous avons soumis les données structurales à une ACP. La figure 6 montre que les deux premiers axes expliquent $74,28 \%$ de la variabilité totale. L'axe 1 explique 49,14\% de cette variabilité. Les variables qui contribuent à 
cet axe sont la strate 4 à $8 \mathrm{~m}$ et la strate 2 à $4 \mathrm{~m}$. Pour le second axe il est défini par les strates inférieure à $2 \mathrm{~m}, 8$ à $16 \mathrm{~m}, 16$ à $32 \mathrm{~m}$ et supérieur à $32 \mathrm{~m}$. Ils expliquent $25,14 \%$.

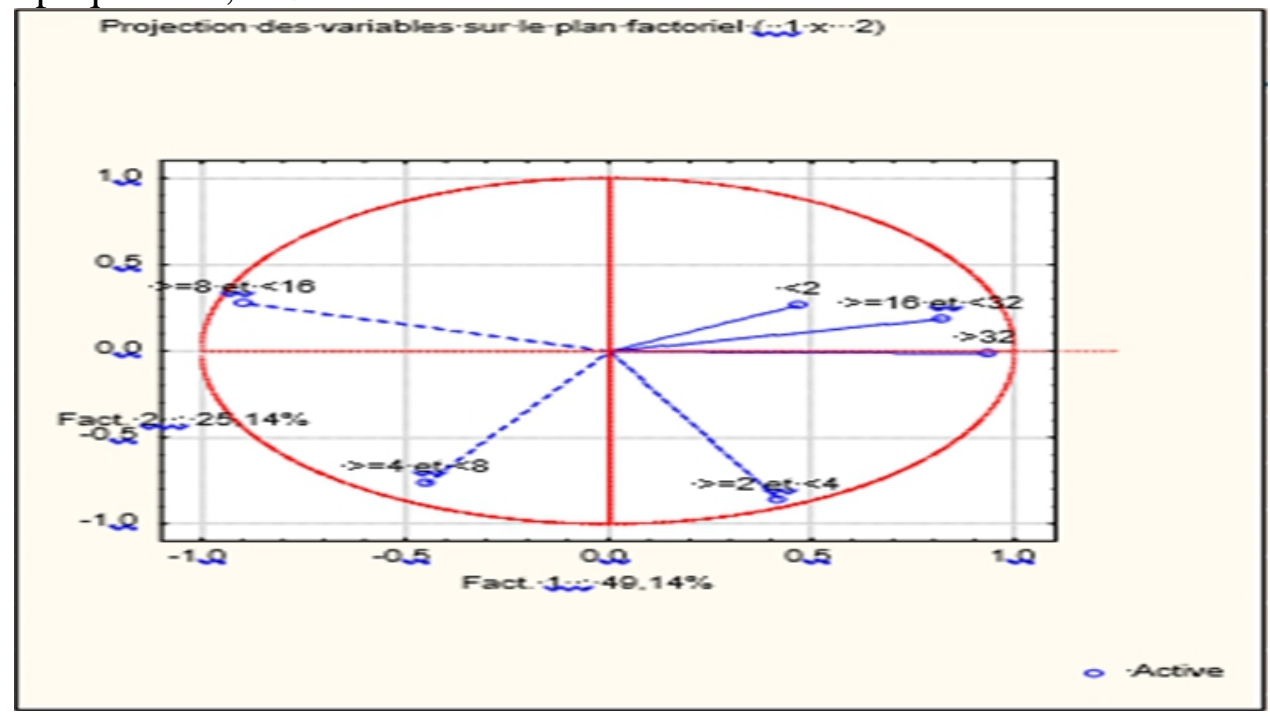

Figure 6: Graphique de corrélation entre les descripteurs structuraux étudiés et l'axe 1et 2 de l'ACP

La classification hiérarchique nous a permis d'obtenir trois groupes de recouvrements structurellement semblables.

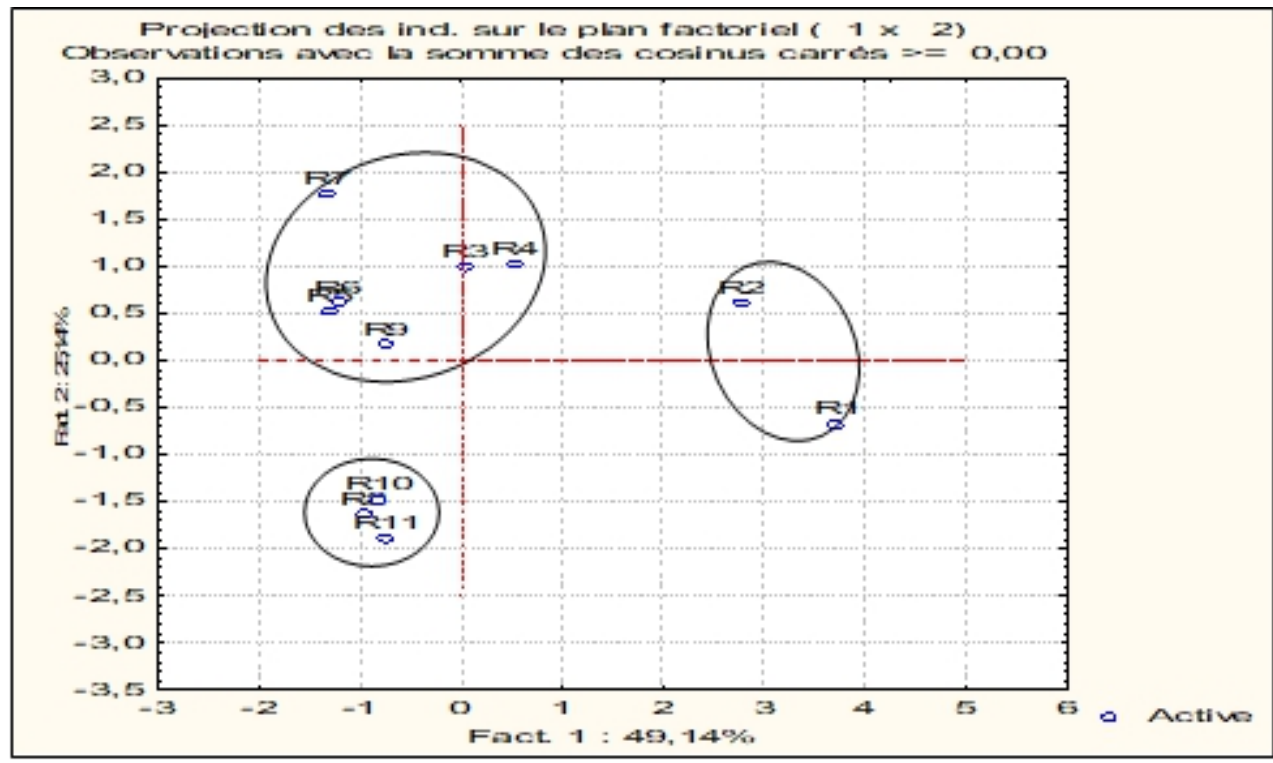

Figure 7: Analyse en composante principale montrant le regroupement des 11 relevés linéaires en trois groups

Le premier groupe comprend les relevés 3, 4, 5, 6, 7 et 9. Les relevés composant ce groupe 1 sont caractérisés par des pourcentages élevés allant de 
66 à $91 \%$ dans la strate moyenne de 8 à 16 m. Par contre aucune espèce n'est obtenue dans la strate supérieure à $32 \mathrm{~m}$. le sous-bois est peu abondant. Les espèces les plus abondantes sont: Afzelia africana, Albizia adianthifolia, Baphia nitida, Berlinia grandiflora, Canthium hispidum, Cassia siamea, Ceiba pentandra, Cola cordifolia, Cremaspora triflora, Dialium dinklagei, Diospyros ferrea, Erythroxyllum emarginatum, Funtumia africana, Geophila repens, Landolphia calabarica, Landolphia togolana, Olyra latifolia, Pouteria alnifolia, Psychotria obscura, Trichilia monadelpha, Turraea heterophylla et Uvaria ovata.

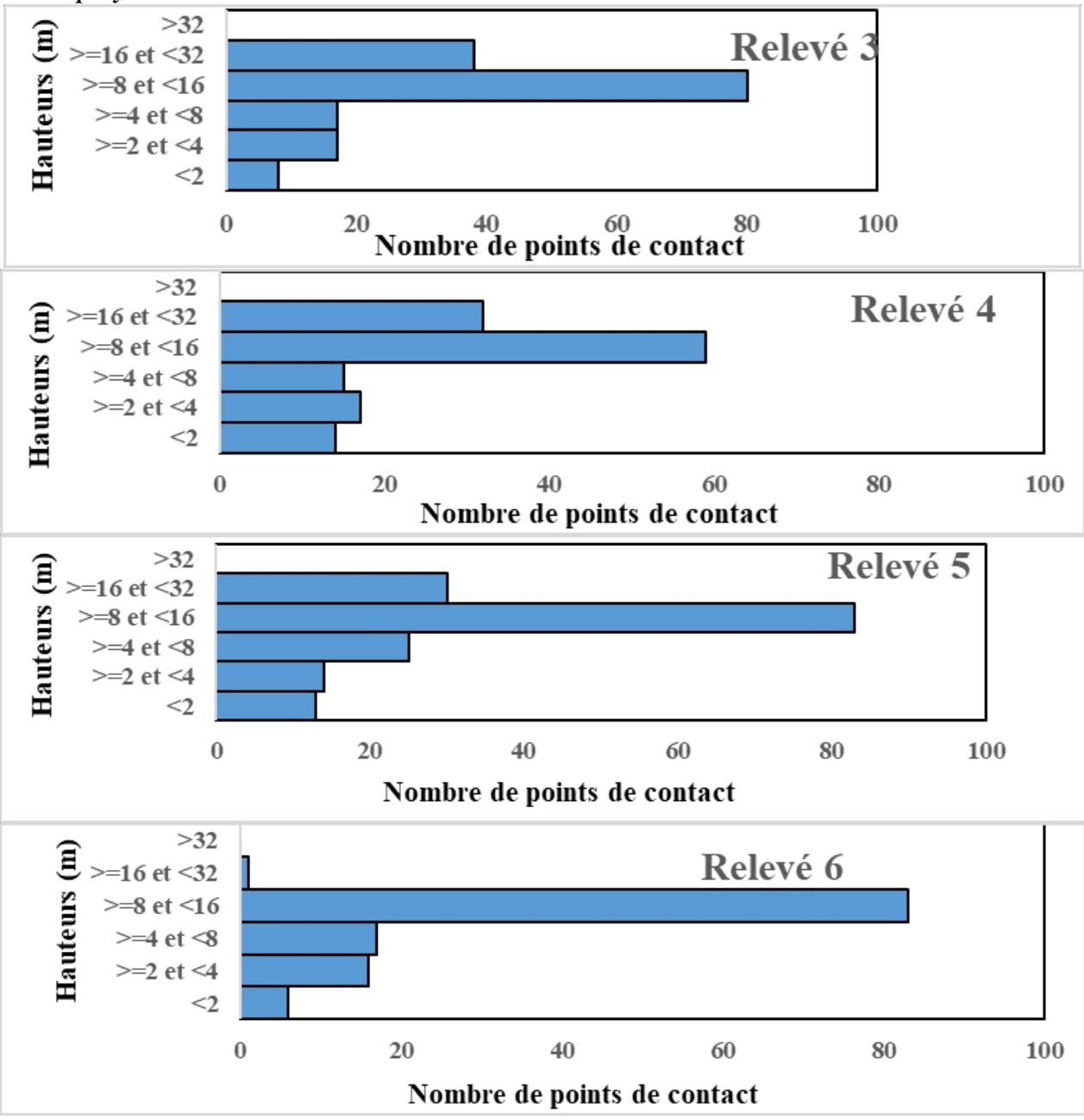




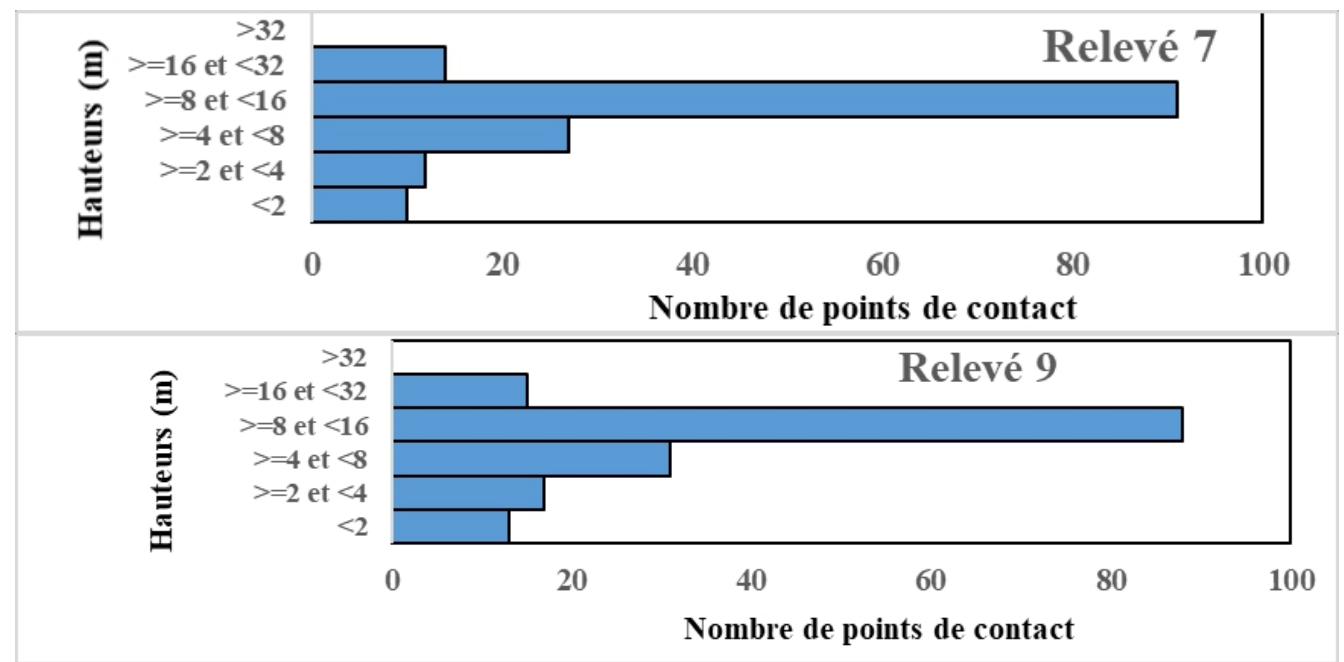

Figure 8: Histogrammes de recouvrements du groupe 1

Le deuxième groupe concerne les relevés 8,10 et 11 . Ces relevés sont caractérisés par un faible pourcentage dans la strate 16 à $32 \mathrm{~m}$. ces relevés sont peu riches en espèces de sous-bois. On observe également une absence totale des espèces émergentes dans la strate supérieure à $32 \mathrm{~m}$. Afzelia africana, Agelaea pentagyna, Bernilia grandiflora, Canthium hispidum, Ceiba pentandra, Cola cordifolia, Cremaspora triflora, Diospyros ferrea, Elaeis guineensis, Erythroxyllum emarginatum, Funtumia africana, Geophila repens, Olax subscorpioidea, Psychotria obscura, Rytyginia canthioides, Smeathmannia et Usteria guineensis possèdent un nombre important d'individus dans ce groupe.

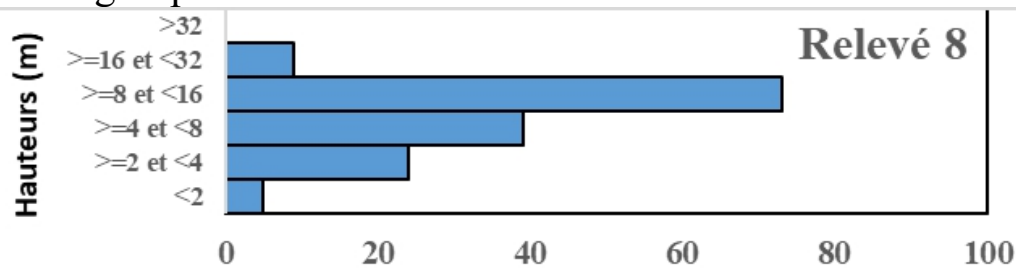

Nombre de points de contact
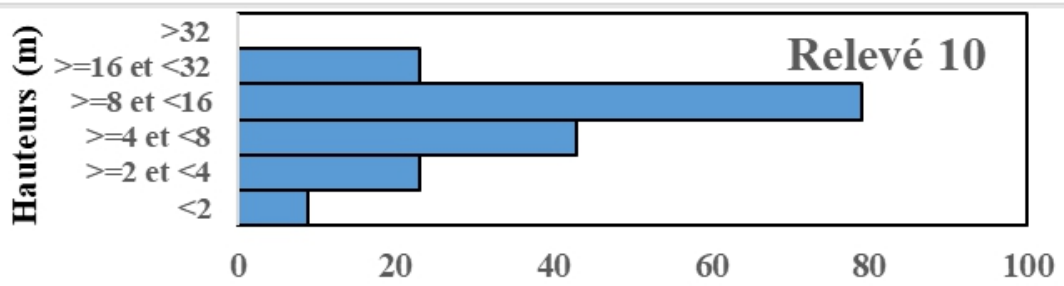

Nombre de points de contact 


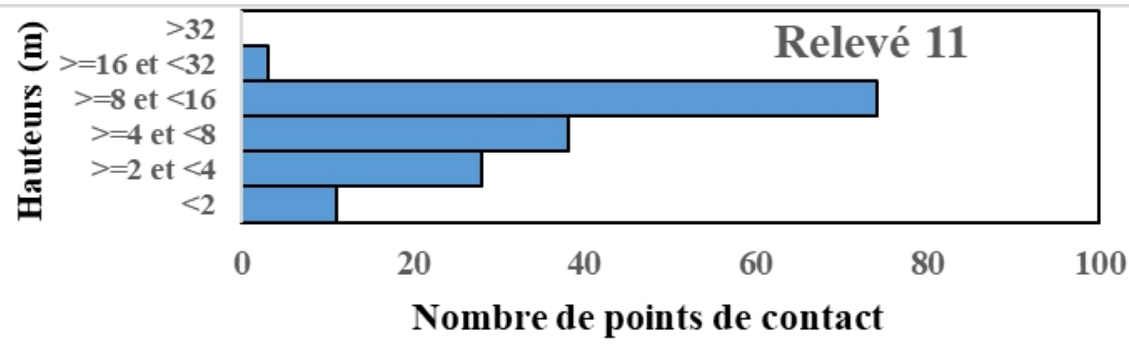

Figure 9: Histogrammes de recouvrements du groupe 2

Les relevés du groupe 3 composés des relevés 1 et 2 se caractérisent par une faible proportion d'espèces dans la strate des émergents $(>32 \mathrm{~m})$ avec une forte proportion dans la strate 16 à $32 \mathrm{~m}$ pour le relevé 1 et dans la strate 8 à $16 \mathrm{~m}$ pour le relevé 2 . Il faut toutefois noter que le nombre d'espèces dans la strate des émergents ( $>32 \mathrm{~m}$ ) est peu supérieure au niveau du relevé 1 par rapport à celle du relevé 2. Afzelia africana, Albizia lebbeck, Baphia nitida, Berlinia grandiflora, Cola caricifolia, Geophila repens, Landolphia togolana, Strophanthus sarmentosus, Trichilia monadelpha, Turraea heterophylla et Uvaria afzelii ont été rencontrées abondamment dans ce groupe.

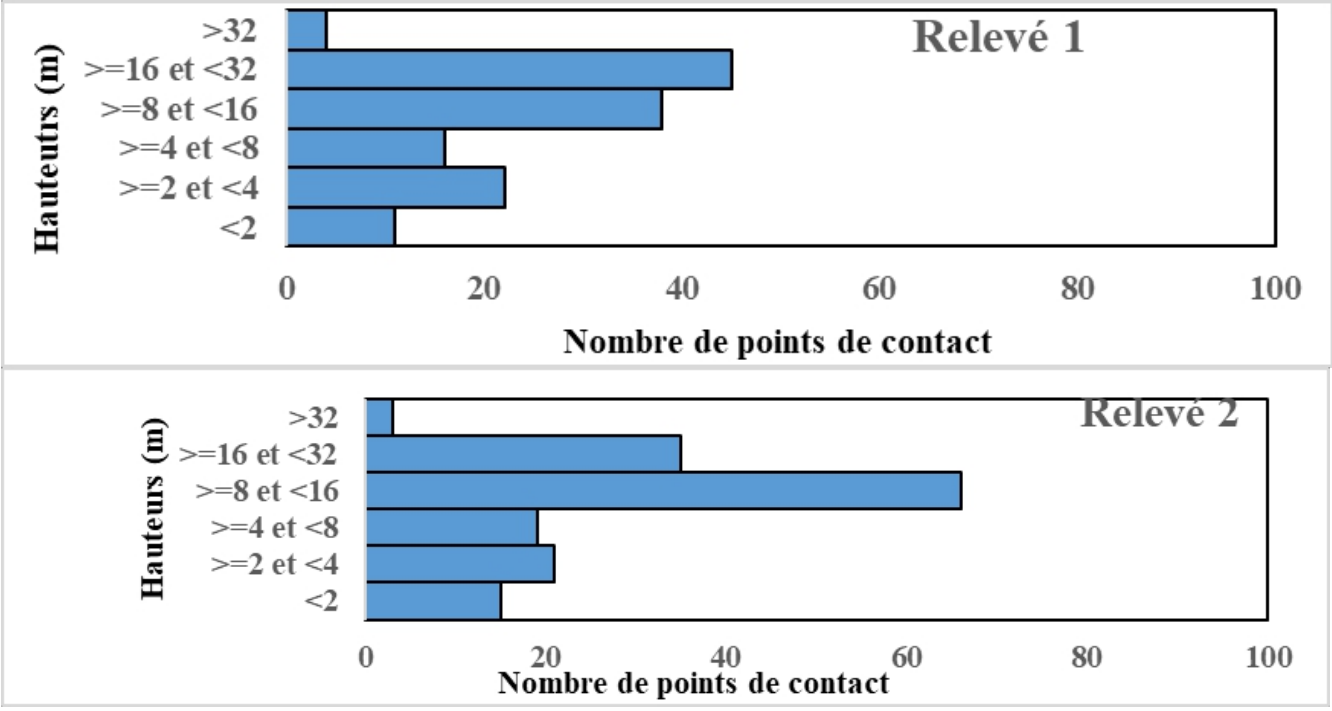

Figure 10: Histogrammes de recouvrements du groupe 3

\section{Discussion}

\section{Etude floristique}

Cette étude a permis d'établir une liste de 176 espèces pour la SPF, reparties en 143 genres et 49 familles contre 115 espèces inventoriées par Devineau et al., (1984). Malgré la faible surface échantillonnée dans cette étude (400 $\mathrm{m}^{2}$ contre $2500 \mathrm{~m}^{2}$ selon ces auteurs), le nombre d'espèces reste élevé. Cette situation pourrait s'expliquer par la combinaison de trois 
différentes méthodes d'échantillonnage. Selon Vroh et al., (2010), l'association de plusieurs méthodes d'inventaire permet d'obtenir des listes floristiques plus riches, même quand les superficies sont moins importantes. La plupart des travaux réalisés dans les forêts tropicales (Bakayoko et al., 2001; Missa et al., 2015) montrent que les Leguminosae dominent toujours les Rubiaceae qui s'expliqueraient par le fait que les Leguminosae regroupent à la fois les Mimosoideae, les Caesalpinoideae et les Fabiodeae qui sont les trois grandes sous-familles (Dibi et al., 2008). Bakayoko et al., (2001) ont montré que les Apocynaceae et les Rubiaceae sont généralement les familles les abondantes en nombre d'espèces dans les forêts denses humides. Ces familles citées sont les plus abondantes en espèces selon notre inventaire. Cela pourrait s'expliquer par le fait que la savane est transformée en forêt.

La présente étude révèle que 30 espèces sont communes à celles de Devineau et al., (1984). Cela pourrait s'expliquer par le fait que ces espèces sont considérées les plus abondantes et les plus diversifiées de la flore de la SPF. De même 47 espèces ne figurent également pas sur la liste de ces mêmes auteurs La découverte de ces nouvelles espèces rencontrées pourrait s'expliquer par la transformation du milieu due à la dissémination des graines soit par les animaux, soit par l'eau où le vent. Par contre, 85 espèces inventoriées par ces auteurs ne figurent pas dans notre inventaire actuel. La disparition où la rareté de ces espèces pourrait s'expliquer soit par l'effort d'échantillonnage soit par une compétition entre la flore humide et la flore sèche ou encore par des facteurs écologiques (pédologie, climat humide, etc) pouvant entrainer leur disparition.

Selon Vuattoux (1970), il a observé l'évolution des ligneux dans la SPF durant quatre ans à Lamto au cours de ces travaux. Il a constaté que le nombre total d'espèces ligneuses passe de 26 en savane brûlée à 35 en SPF. Le nombre de pionnières forestières passe de 11 à 20 espèces. Lors de la présente étude nous avons pu recenser 54 espèces forestières largement supérieure à celles obtenues par Vuattoux. Cela pourrait s'expliquer par la transformation du milieu au profit de la forêt et aux dépens de la savane. Spichiger, (1975), a inventorié 25 espèces phanérophytes forestières contre 54 espèces selon notre étude. Cette supériorité en nombre d'espèces pourrait s'expliquer par une régression de la savane au profit de la forêt.

Notre inventaire révèle que sur 11 espèces reconnues comme exploitables en tant que bois d'œuvre, on compte 10 espèces forestières, signe de la transformation de la savane en forêt. Cette richesse de la flore de la SPF en essences exploitables représente une raison supplémentaire pour l'accroissement de sa surveillance afin d'éviter les exploitations forestières clandestines (Dibi et al., 2008).

La flore de cette SPF est dominée par des taxons des régions GuinéoCongolaises et Guinéo-Congolaises et Soudano-Zambiennes. Cette richesse 
en nombres d'espèces à statut particulier pourrait s'expliquer par la situation géographique de Lamto. En fait, cette Réserve Scientifique serait proche des zones Guinéennes, Soudaniennes et Zambiennes. Cela confirme l'appartenance de cette réserve à la zone de transition forêt-savane comme c'est le cas d'autres aires protégées telles que le Parc National de la Marahoué. De même la valeur de la SPF pour la conservation de la biodiversité est déterminée à travers la présence de plusieurs espèces à statut particulier: espèces endémiques, vulnérables, à préoccupation mineure, à donnée insuffisante et quasi menacée (IUCN, 2018). Cette situation serait dû au fait que l'écotone forêt-savane de la réserve de Lamto est localisée dans la zone d'endémisme (hotspot) de la Côte d'Ivoire (Konaté \& Kampmann, 2010).

Les spectres morphologiques et biologiques sont caractérisés par une importante proportion d'arbustes et de lianes mésophanérophytes. La forte proportion des ligneux et des lianes témoigne de la bonne reconstitution des peuplements et cela pourrait s'expliquer par la forte quantité de litière qui enrichi le sol et l'absence de pressions anthropiques.

La valeur moyenne de l'indice de diversité de Shannon-Weaver obtenue au cours de cette étude obtenue est de 1,217 contre 5,772 obtenu par Dibi et al., (2008). Cette valeur de cet indice que nous avons obtenu reste inférieure à celle de ces mêmes auteurs. De même l'indice d'équitabilité moyen de cette étude est de 0,765 contre 0,951 . Cette valeur reste toujours inférieure à celle de Dibi et al., (2008). Cette situation pourrait s'expliquer par le fait que l'absence des feux expliquerait la faible équitabilité de la répartition des espèces due à une faible installation d'espèces ligneuses.

\section{Structure de la végétation}

La classification hiérarchique ascendante montre trois groupes floristiques. Cette classification pourrait donc, avant tout, représentée un effet d'échantillonnage entre relevé mené dans cette formation de structure semblable. Les études basées sur la méthode linéaire telle que mentionnée dans cette étude dans la SPF est l'une de son genre. Cela limite donc nos comparaisons à cette seule étude.

L'interprétation des graphiques de recouvrement renseigne sur la structure verticale des différents relevés. On remarque que ces graphiques sont différents les uns des autres. La densité des individus constatée sur les histogrammes de recouvrement dans presque tous les relevés montre un pourcentage élevé de contacts dans la strate 8 à $16 \mathrm{~m}$. L'absence des feux dans cette formation a pu, en effet, favoriser la repousse d'espèces telles que les arbustes qui y trouvent les conditions idéales. La forte proportion d'espèces lianescentes dans la strate inférieure et l'existence d'une strate arbustive dense dans la strate moyenne s'expliqueraient par la diversité floristique de ces espèces. Cette situation pourrait aussi s'expliquer par une régénération 
naturelle de la Savane Protégée des Feux. Plusieurs travaux ont montré la prolifération de ces catégories d'espèces après perturbation anthropique (Guariguata \& Ostrertag, 2001 ; Chinea \& Helmer, 2003).

Très peu d'individus de plus de $32 \mathrm{~m}$ de hauteur ont été observés au niveau des différents relevés. Les quelques individus de taille supérieure à cette hauteur n'ont été rencontrés que sur les relevés 1 et 2 . Ceci s'expliquerait par l'émergence au-dessus de la canopée de certaines espèces comme Ceiba pentandra et Berlinia grandiflora. La faible présence d'individus de grande taille sur ces relevés, est sans doute liée aux activités antérieures des pratiques des feux qui ont eu lieu dans pratiquement toute la savane.

\section{Conclusion}

La Savane Protégée des Feux (SPF) comprend 176 espèces reparties entre 143 genres et 49 familles. Les familles les plus rencontrées dans l'ensemble sont les Rubiaceae, les Leguminosae et les Apocynaceae. La Savane Protégée des Feux présente une grande valeur écologique avec la présence des espèces à statut particulier. Cette étude a donc permis de montrer que la composition floristique et la structure de la SPF, sont influencées par l'implantation des espèces forestières exploitables ou exploitées (11 espèces).

La structure de la végétation relève 75 à $80 \%$ d'espèces de recouvrement. Des baisses d'espèces sont toutefois observées dans la strate 16 à $32 \mathrm{~m}$ dans la quasi-totalité des relevés avec une hétérogénéité, entre les strates intermédiaires.

Cette étude affirme que la végétation évolue au profit de la forêt et aux dépens de la savane. La flore sèche est en régression vis-à-vis de la flore humide. Les relevés linéaires ont montré des variations floristiques différentes de celles des forêts originelles.

\section{Remerciements}

Nous remercions le Docteur Yéo KOLO responsable de la station d'écologie de Lamto qui nous a permis de réaliser nos travaux de terrain au sein de ladite institution. Les auteurs de cet article voudraient traduire leur reconnaissance au Centre Suisse de Recherches Scientifiques (CSRS), qui a contribué à l'identification des espèces végétales inventoriées.

\section{References:}

1. Adou, C.Y., Bakayoko, A., Akpatou, K.B \& N'guessan, K. (2011). Impacts de pressions anthropiques sur la flore et la structure de la végétation dans la forêt classée de Monogaga, Côte d'Ivoire. Journal of Animal \& Plant Sciences, 12(2) ; 1560-1572.

2. Aké-Assi, L. (2002). "Flore de la Côte d'Ivoire: catalogue systématique, biogéographie et écologie". Volume 2: Mémoire de 
Botanique systématique. Conservatoire et Jardin Botanique de Genève; Boisseria 58, 441 p.

3. APG IV. (2016). An update of the Angiosperm Phylogeny Group classification for the orders and families of flowering plants. Bot. $J$. Linn. Soc. 161: 105-121p.

4. Bakayoko, A. (1999). Comparaison de la composition floristique et de la structure forestière de parcelle de la Forêt Classée de Bossématié dans l'Est de la Côte d'Ivoire. Mémoire DEA Ecologie Tropicale, U.F.R. Biosciences, Université de Cocody-Abidjan, 72 pages.

5. Bakayoko, A., Kouamé, N.F., Tra-Bi, F.H. \& Traoré, D. (2001). Quelques aspects floristiques et structuraux de la forêt classée de Bossématié, dans l'Est de la Côte d'Ivoire. Ann. Bot. Afr. O. (O), pages 7-19.

6. Barbault, R. (1982). Ecologie des populations et des peuplements, Paris. 200p

7. Bond, W.J \& Parr, C.L. (2010). Beyond the forest edge : Ecology, diversity and conservation of the grassy biomes. Biological conservation, vol. 143, p. 2395-2404.

8. Brunelle, A., Minclkey, T.A., Delgadillo, J \& Blissett, S. (2013). A longterm perspective on woody plant encroachment in the desert southwest, New Mexico, USA. Journal of Vegetation Science, doi: 10.1111/jvs.12125 (consultée le 28 décembre 2015).

9. César, J \& Menaut, J. C. (1974). Analyse d'un écosystème tropical humide: la savane de Lamto, 2, Le peuplement végétal. Bulletin de Liaison Chercheurs Lamto $\mathrm{N}^{\circ}$ spécial, 2: 161p. Côte d'Ivoire). Thèse de 3e cycle, Univ. Paris, $95 \mathrm{p}$.

10. Chatelain, C. (1996). Possibilités d'Application de l'Imagerie Satellitaire à Haute Résolution pour l'étude des Transformations de la végétation en Côte d'Ivoire Forestière. Thèse de Doctorat, Univ. Genève 206p

11. Chinea, J D \& Eileen, H. H. (2003). Diversity and composition of tropical secondary forests recovering from large-scale clearing: results from the 1990 inventory in Puerto Rico. Forest Ecology and Management 180:227240

12. Delmas, J. (1967). Recherches écologiques dans la savane de Lamto: premier aperçu sur les sols et leur valeur agronomique, La Terre et la Vie 3, 216-227p.

13. Demers-Lemay M. (2014). Utilisation du feu par la communauté Guarani Nandeva de Pykasu dans les écosystèmes semi-arides du Chaco Boréal, Département de Boquerón, Paraguay. Rapport de stage, Université de Sherbrooke, Sherbrooke, 67 p. 
14. Devineau, J.L. (1975). "Etude quantitative des forets galeries de Lamto (moyenne Côte d'Ivoire)", Thèse de Doctorat de $3^{\text {ème }}$ cycle de l'université de Paris VI, $190 \mathrm{p}$.

15. Devineau, J.L., Lecordier, C \& Vuattoux, R. (1984). Evolution de la diversité spécifique du peuplement ligneux dans une succession préforestière de colonisation d'une savane protégée des feux (Lamto, Côte-d'Ivoire) Candollea 39: 104-134 p

16. Dibi, H., Adou, Y C., N'guessan, K. E., Koné, M \& Sagne, Y .C. (2008). Analyse de la diversité floristique du parc national de la Marahoué, Centre-Ouest de la Côte d'Ivoire, Afrique science, 4 (03), pp. 552-557

17. Eldridge, D.J., Bowker, M.A., Maestre, F.T., Roger. E., Reynolds, J.F \& Whitford. W.G. (2011). Impacts of shrub encroachment on ecosystem structure and functioning : towards a global synthesis. Ecology Letters, vol. 14, p. 709-722.

18. Estell, R., Havstad, K.M., Cibils, A.F., Fredrickson, E.L., Anderson, D.M., Schrader, T.S \& James, D.K. (2012). Increasing shrub use by livestock in a world with less grass. Rangeland Ecology et Management, vol. 65, p. 553-562.experience and economy of conservation areas?' Koedoe, vol. 55, $\mathrm{n}^{\circ}$. 1, p. 1-9.

19. Gautier, L., Chatelain, C. V. \& R, Spichiger. (1994). Presentation of a releve for vegetation studies based on high resolution satellite imagery. In : Comptes rendus de la treizième réunion plénière de l'AETFAT, Zomba, Malawi. Nat. Herb. Bot. Gard. Malawi. Vol. 2 : 1339-1350.

20. Guariguata, M. R \& Ostrertag, R. (2001). Neotropical secondary forest succession: changes in structural and functional characteristics. Forest Ecology and Management 148:185206

21. Gueulou, N., Ouattara, N.D., Konan, D., Gnahoré, E., Missa, K \& Bakayoko, A. (2018). Diversité floristique et structurale de la forêt galerie du Bandama dans la Réserve Scientifique de Lamto en Côte d'Ivoire. Afrique SCIENCE 14(4) (2018)- 452439

22. Hutchinson, J \& Dalziel, J. M. (1954). Flora of west tropical Africa. Crown Agent, London, 3 : 544-828.

23. Konaté, S \& Kampmann, D. (2010). "Atlas de la Biodiversité de l'Afrique de_l'Ouest", Tome III : Côte d'Ivoire, Abidjan et Frankfurt/Main. (eds) (2010)

24. Menaut J. C. (1983). The vegetation of African savannas, in Bourlire F., editor, Tropical Savannas, Elsevier, Amsterdam, p. 109-149.

25. Missa, K., Ouattara, D.N., Koné, M \& Bakayoko, A., (2015) : Etude floristique et diversité de la forêt des Marais Tanoé-Ehy (Sud- Est de la Côte d'Ivoire). Journal of Animal \&Plant Sciences, 2015. Vol.25, Issue 3: 3917-3938 
26. Pagney, P., (1988). Le climat de Lamto (Côte d'Ivoire) in Lamotte M. et Tirefort J.L. (Eds), Le climat de la savane de Lamto (Côte d'Ivoire) et sa place dans les climats de l'ouest africain. Travaux Chercheurs de Lamto: 31-79p.

27. Piélou, E. C. (1966). Species-diversity and pattern-diversity in the study of ecological succession. Journal of Theoretical Biology 10: 370-383p

28. Riou, G. (1964). Note sur les sols complexes des savanes préforestières en Côte d' Ivoire. Ann. Univ. Abidjan, sér. Lettres et Sciences Humaines, 1, p. 17-35.

29. Shannon, C \& Weaver, W. (1949). A mathematical theory of commuty, University of Illinois, pres Urbana, 117p.

30. Spichiger, R. (1975). Contribution à l'étude du contact entre flores sèche et humide sur les lisières des formations forestières humides semi-décidues du V baoulé et de son extension nord-ouest (Côte d'Ivoire centrale). Thèse Univ. Genève $261 p$

31. IUCN., (2018). Red List of Threatened Species. http://www.iucnredlist. org/. (consulté le 15 septembre 2018)

32. UICN/BRAO., (2008). Evaluation de l'efficacité de la gestion des aires protégées : parcs et réserves de Côte d'Ivoire 13p

33. Vroh, B.T.A., Adou Y.C.Y., Kouamé, D., N'da, D.H. \& N'guessan, K.E. (2010). Diversités Floristique et Structurale sur le Site d'une Réserve Naturelle Volontaire à Azaguié, Sud-est de la Côte d'Ivoire. European Journal of Scientific Research 45 (3): 411-421.

34. Vuattoux, R. (1970). Observations sur l'évolution des strates arborée et arbustive dans la savane de Lamto (Côte d'Ivoire). Annales de l'Université d'Abidjan, Série E, 3 (1), 285-315p.

35. Vuattoux, R., Konaté, S., Abbadie, L., Barot, S., Gignoux, J \& Lahoreau, G. E. (1998). History of the Lamto Ecology Station and Ecological Studies at Lamto 1-2p. 